\title{
Alignment in TKA: what has been clear is not anymore!
}

\author{
Michael T. Hirschmann ${ }^{1,2}$ (D) Roland Becker $^{3} \cdot$ Reha Tandogan $^{4} \cdot$ Pascal-André Vendittoli $^{5} \cdot$ Stephen Howell ${ }^{6}$
}

Received: 21 May 2019 / Accepted: 6 June 2019 / Published online: 12 June 2019

(c) European Society of Sports Traumatology, Knee Surgery, Arthroscopy (ESSKA) 2019

The optimal implant orientation when performing a total knee arthroplasty (TKA) remains a timely, pertinent, and unanswered question. For many years, the alignment debate has filled journals and congresses, and monopolised discussions among knee surgeons all around the globe. In the development of TKA surgery, Michael Freeman introduced the concept of right-angled femoral and tibial bone cuts (mechanical alignment) and the idea of parallel and equal flexion and extension spaces. Using the mechanical alignment target, the knee surgeon strived to create a neutral lower limb alignment represented by a hip-knee-ankle angle target of $180^{\circ} \pm 3^{\circ}[4,12]$. Although the mean hip-kneeankle angle (HKA) of patients scheduled for TKA is near neutral, there is a wide variation and only $0.1 \%$ have neutral femoral and tibial mechanical axes [1]. With a systematic approach, mechanical alignment introduces anatomic modifications for many individuals and results in unequal medial-lateral or flexion-extension bone resections. Multiple ligament release techniques and algorithms have been proposed to re-balance the unbalanced gaps created.

In the past years we have seen increased questioning of the concept of mechanical alignment, which has been considered the gold standard for decades. Historically, the alignment philosophy for TKA was driven by the desire to

Michael T. Hirschmann

michael.hirschmann@ksbl.ch;

michael.hirschmann@unibas.ch

1 Department of Orthopaedic Surgery and Traumatology, Kantonsspital Baselland (Bruderholz, Liestal, Laufen), 4101 Bruderholz, Switzerland

2 University of Basel, Basel, Switzerland

3 Department of Orthopaedics and Traumatology, Brandenburg Medical School Theodore Fontane, 14770 Brandenburg, Germany

4 Ortoklinik and Cankaya Orthopedics, Ankara, Turkey

5 Department of Surgery, Maisonneuve-Rosemont Hospital, Montreal University, Montreal, QC, Canada

6 Professor of Biomedical Engineering, University of California at Davis, Davis, USA maximize durability and relieve pain with less regard for restoring normal knee kinematics and function. However, several landmark studies have shown the concept of neutrally aligning every TKA is dogma and not true anymore [23].

Questioning such a dogma leads to a certain amount of uncertainty among knee surgeons and opens the door to non-scientific subjective definitions and personal opinions of what alignment targets are preferred and which ones to safely recommend. Hence, it is important to unambiguously describe and define the current implant orientation and alignment options [18]. Only when knee surgeons use the same definitions to discuss alignment progress can be made and misinterpretation be limited.

Anatomical alignment was introduced in the 1980s by Hungerford and Krackow with the goal to improve functionality by closer mimicking the native knee alignment [18]. With a systematic approach, anatomical alignment still aims for a neutral HKA, but the bones are cut $3^{\circ}$ oblique to their mechanical axes to reflect the population's mean native joint line orientation $\left(3^{\circ}\right.$ femoral valgus and $3^{\circ}$ tibial varus) [18].

Kinematic alignment, first proposed in 2006 by Howell et al., is an 'individualised' or patient-specific technique, aiming to restore the pre-arthritic or native limb and joint line alignment of each patient $[3,8,9,11]$. By resurfacing the knee joint, kinematic alignment technique aims to coalign the axes and joint lines of the components with the three 'kinematic' axes and joint lines of the pre-arthritic or native knee. Femoral and tibial bone resection thicknesses checked with caliper measurements should match the thickness of the components after compensating for wear and the kerf of the saw cut. Intrinsically, it preserves/restores native ligament laxities, does not create gap imbalance and thus minimises the need for ligament release [14]. In his protocol, Howell does not place restrictions on the patient's anatomy and post-operative correction. Kinematic alignment requires a precise surgical technique which can be performed by different techniques: manual instruments, computer navigation, personalised instruments, and computer guidance, 
with caliper verification that the executed resections are correct $[10,14]$.

Some knee anatomies may be inherently biomechanically inferior, or may have been altered by metabolic bone disease, childhood deformity, etc. Concerns remain about restoring severe patho-anatomies, which may not be compatible with current TKA prostheses and fixation methods [9, 22]. Keeping in mind these uncertainties, Vendittoli et al. recommended "safe zones" for TKA alignment and suggested the use of a restricted kinematic alignment protocol [1]. The algorithm involves modifications of bony cuts within a "safe range" defined by the following criteria: independent tibial and femoral cuts must be within $\pm 5^{\circ}$ of the mechanical axis of the respective bone and the overall resulting hip-kneeankle angle (HKA) must fall within $\pm 3^{\circ}$ of neutral.

However, these concerns about the need to restrict the degree of preoperative deformity and post-operative correction when performing kinematically aligned TKA are not supported by available knowledge. In a mid-term study, of unrestricted kinematic alignment the 10-year implant survival (i.e., $1.5 \%$ revised for aseptic reasons) and yearly revision rate (i.e., $0.3 \%$ ) met the expected gold standard of mechanically aligned TKA, and the 2 to 9 -year incidence of tibial component failure was negligible [9, 13]. Despite restoring a more varus limb alignment, kinematic alignment, in gait analyses, produced a lower knee adduction moment and medial tibial compartment load and more normal gait than mechanical alignment $[2,15]$. The intra-operative forces in the medial and lateral compartments of patients with outlier alignment of the limb, knee, and tibia are comparable with those with in-range alignment, with no evidence of overload of the tibial compartments [19, 20, 22]. Accurate restoration of the distal femoral, posterior femoral, and tibial joint lines within $\pm 1 \mathrm{~mm}$ is needed as deviations as small as $2 \mathrm{~mm}$ and $2^{\circ}$ increase tibial compartment forces beyond those of the native knee, which patients may perceive as stiffness or limited motion [16, 17, 21].

The latest and most compelling support for use of kinematic or an 'individualised' alignment philosophy in place of mechanical alignment is from the systematic classification of the phenotype of the native limb and knee joint line by Hirschmann et al. [5-7]. Guided by the individual phenotype identified the optimal alignment for each knee is found. It is about a more meticulous planning in 3D and the decision if an off the shelf knee could do it or if the knee needs a customised TKA.

Due to the significant deficiencies in both our knowledge and technology in the past, we were far from replicating normal knee kinematics with TKA. Current limitations in TKA function and patient satisfaction should stimulate us to question our practice. Implant design and surgical techniques need to be advanced to better reproduce the anatomy and kinematics of native knees and ultimately provide a forgotten joint. As with many things in life there are different phases of adoption of a newer alignment philosophy such as kinematic or individualised alignment. The surgeons that are early adopters are on the forefront of change and their concepts need examination and vetting. The late adopters are conservative and remain critical until the newer alignment philosophy is proven superior to mechanical alignment. The mass in between, the middle adopters, remain rather indecisive and somewhat confused about the definition and benefits and short-comings of available alignment philosophies. In one of our previously published papers the authors stated they used kinematic alignment; however, their surgical method was challenged as not meeting the definition of kinematic alignment in a letter to the editor by Riviere et al. The correction will be published in this issue.

It appears that what was clear for decades is not so clear anymore. The discussion needs to go on and will go on. Our understanding will be enhanced by the use of an unambiguous definition of alignment by those that report outcomes of TKA.

\section{References}

1. Almaawi AM, Hutt JRB, Masse V, Lavigne M, Vendittoli PA (2017) The impact of mechanical and restricted kinematic alignment on knee anatomy in total knee arthroplasty. J Arthroplast 32(7):2133-2140

2. Blakeney W, Clement J, Desmeules F, Hagemeister N, Riviere C, Vendittoli PA (2018) Kinematic alignment in total knee arthroplasty better reproduces normal gait than mechanical alignment. Knee Surg Sports Traumatol Arthrosc. https://doi.org/10.1007/ s00167-018-5174-1

3. Gu Y, Roth JD, Howell SM, Hull ML (2014) How frequently do four methods for mechanically aligning a total knee arthroplasty cause collateral ligament imbalance and change alignment from normal in white patients? AAOS exhibit selection. J Bone Joint Surg Am 96(12):e101

4. Hess S, Moser LB, Amsler F, Behrend H, Hirschmann MT (2019) Highly variable coronal tibial and femoral alignment in osteoarthritic knees: a systematic review. Knee Surg Sports Traumatol Arthrosc. https://doi.org/10.1007/s00167-019-05506-2

5. Hirschmann MT, Hess S, Behrend H, Amsler F, Leclercq V, Moser LB (2019) Phenotyping of hip-knee-ankle angle in young non-osteoarthritic knees provides better understanding of native alignment variability. Knee Surg Sports Traumatol Arthrosc. https ://doi.org/10.1007/s00167-019-05507-1

6. Hirschmann MT, Moser LB, Amsler F, Behrend H, Leclercq V, Hess $S$ (2019) Phenotyping the knee in young non-osteoarthritic knees shows a wide distribution of femoral and tibial coronal alignment. Knee Surg Sports Traumatol Arthrosc. https://doi. org/10.1007/s00167-019-05508-0

7. Hirschmann MT, Moser LB, Amsler F, Behrend H, Leclerq V, Hess S (2019) Functional knee phenotypes: a novel classification for phenotyping the coronal lower limb alignment based on the native alignment in young non-osteoarthritic patients. Knee Surg Sports Traumatol Arthrosc. https://doi.org/10.1007/s00167-01905509-z 
8. Howell SM, Howell SJ, Kuznik KT, Cohen J, Hull ML (2013) Does a kinematically aligned total knee arthroplasty restore function without failure regardless of alignment category? Clin Orthop Relat Res 471(3): 1000-1007

9. Howell SM, Shelton TJ, Hull ML (2018) Implant survival and function 10 Years after kinematically aligned total knee arthroplasty. J Arthroplasty 33(12):3678-3684

10. Hutt JR, LeBlanc MA, Masse V, Lavigne M, Vendittoli PA (2016) Kinematic TKA using navigation: surgical technique and initial results. Orthop Traumatol Surg Res 102(1):99-104

11. Lee YS, Howell SM, Won YY, Lee OS, Lee SH, Vahedi H, Teo SH (2017) Kinematic alignment is a possible alternative to mechanical alignment in total knee arthroplasty. Knee Surg Sports Traumatol Arthrosc 25(11):3467-3479

12. Moser LB, Hess S, Amsler F, Behrend H, Hirschmann MT (2019) Native non-osteoarthritic knees have a highly variable coronal alignment: a systematic review. Knee Surg Sports Traumatol Arthrosc. https://doi.org/10.1007/s00167-019-05417-2

13. Nedopil AJ, Howell SM, Hull ML (2017) What mechanisms are associated with tibial component failure after kinematicallyaligned total knee arthroplasty? Int Orthop 41(8):1561-1569

14. Nedopil AJ, Singh AK, Howell SM, Hull ML (2018) Does calipered kinematically aligned TKA restore native left to right symmetry of the lower limb and improve function? J Arthroplasty 33(2):398-406

15. Niki Y, Nagura T, Nagai K, Kobayashi S, Harato K (2018) Kinematically aligned total knee arthroplasty reduces knee adduction moment more than mechanically aligned total knee arthroplasty. Knee Surg Sports Traumatol Arthrosc 26(6):1629-1635

16. Riley J, Roth JD, Howell SM, Hull ML (2018) Increases in tibial force imbalance but not changes in tibiofemoral laxities are caused by varus-valgus malalignment of the femoral component in kinematically aligned TKA. Knee Surg Sports Traumatol Arthrosc 26(11):3238-3248

17. Riley J, Roth JD, Howell SM, Hull ML (2018) Internal-external malalignment of the femoral component in kinematically aligned total knee arthroplasty increases tibial force imbalance but does not change laxities of the tibiofemoral joint. Knee Surg Sports Traumatol Arthrosc 26(6):1618-1628

18. Riviere C, Iranpour F, Auvinet E, Howell S, Vendittoli PA, Cobb J, Parratte S (2017) Alignment options for total knee arthroplasty: a systematic review. Orthop Traumatol Surg Res 103(7):1047-1056

19. Roth JD, Howell SM, Hull ML (2018) Kinematically aligned total knee arthroplasty limits high tibial forces, differences in tibial forces between compartments, and abnormal tibial contact kinematics during passive flexion. Knee Surg Sports Traumatol Arthrosc 26(6):1589-1601

20. Shelton TJ, Howell SM, Hull ML (2019) Is there a force target that predicts early patient-reported outcomes after kinematically aligned TKA? Clin Orthop Relat Res 477(5):1200-1207

21. Shelton TJ, Howell SM, Hull ML (2018) A total knee arthroplasty is stiffer when the intraoperative tibial force is greater than the native knee. J Knee Surg. https://doi.org/10.1055/s-0038-1675421

22. Shelton TJ, Nedopil AJ, Howell SM, Hull ML (2017) Do varus or valgus outliers have higher forces in the medial or lateral compartments than those which are in-range after a kinematically aligned total knee arthroplasty? limb and joint line alignment after kinematically aligned total knee arthroplasty. Bone Joint J 99-B(10):1319-1328

23. Slevin O, Hirschmann A, Schiapparelli FF, Amsler F, Huegli RW, Hirschmann MT (2018) Neutral alignment leads to higher knee society scores after total knee arthroplasty in preoperatively nonvarus patients: a prospective clinical study using 3D-CT. Knee Surg Sports Traumatol Arthrosc 26(6):1602-1609

Publisher's Note Springer Nature remains neutral with regard to jurisdictional claims in published maps and institutional affiliations. 